\title{
Spontaneous self-embedding of three-dimensional SiGe islands
}

\author{
E. Mateeva, ${ }^{\text {a) }}$ P. Sutter, ${ }^{\text {a) }}$ and M. G. Lagally ${ }^{\text {b) }}$ \\ University of Wisconsin-Madison, Madison, Wisconsin 53706
}

(Received 20 July 1998; accepted for publication 20 November 1998)

It is shown that, under appropriate conditions, high-Ge-concentration coherent three-dimensional $\mathrm{SiGe}$ islands grown on $\mathrm{Si}(100)$ self-embed in a matrix of a low-Ge-concentration alloy. The process may be more generally useful for preserving the shape of self-assembled "quantum dot" islands during embedding in a matrix material. (c) 1999 American Institute of Physics.

[S0003-6951(99)05104-9]

The possibilities of self-assembled formation of dense array of islands with uniform and controllable sizes and shapes from several lattice-mismatched material combinations (In, Ga)As/GaAs, InP/(In, Ga)P, SiGe/Si have been studied extensively, as self-assembly may be an elegant way of fabricating nanoscale structures. ${ }^{1-6}$ Lateral confinement of carriers in such "quantum dot" islands is expected to add new functionality to future electronic and optoelectronic devices. The uniformity of island sizes required in such devices has been achieved in multilayer structures having alternating layers of coherently strained $\mathrm{SiGe}$ (InAs) islands and $\mathrm{Si}$ (GaAs) spacer layers. ${ }^{1,2,6}$ In multilayers the size filtering action of the spacer layer produces progressive convergence towards uniformity of island size and spacing. In both $\mathrm{Ge} / \mathrm{Si}$ and InAs/GaAs materials systems the growth of the spacer layer affects the shape and composition of the islands. While for the InAs/GaAs system there is controversy about the final shape of the embedded islands, ${ }^{7}$ the effect of embeddinglayer growth is quite well understood in the $\mathrm{Ge} / \mathrm{Si}$ system. ${ }^{8-10}$ Coherent free-standing SiGe islands assume two well-defined shapes: pyramidal, with shallow $\{105\}$ facets ("huts") and dome shaped, with higher-angle facets ("domes"). ${ }^{11-13}$ Both types of islands change their shape to a truncated mesa bounded on top by a (100) facet during the growth of the $\mathrm{Si}$ layer, over a wide range of growth temperatures. ${ }^{6,8-10}$ The change of shape is accompanied by a reduction of the initial height of the islands, which at high temperatures can be significant enough to transform the islands into a two-dimensional (2D) layer. A real-time study of self-assembled $\mathrm{SiGe}$ islands during embedding-layer growth shows that $\mathrm{Si}$ deposition drives a kinetically determined change in the shape of the islands, making their final shape hard to predict, as it is not a thermodynamically stable one. ${ }^{9,10}$

Critical to the study of the electronic properties of these nanoscale three-dimensional (3D) islands-huts and domes-and their eventual use in devices is that they be embedded in a matrix in a manner that avoids uncontrolled shape changes. We have shown that one possible way of restricting the $3 \mathrm{D}$ island shape change during embedding in $\mathrm{Si}$ is by rapid deposition at low temperatures. ${ }^{10}$ In this letter

${ }^{a)}$ Permanent address: Department of Physics, Colorado School of Mines, Golden, Colorado 80401.

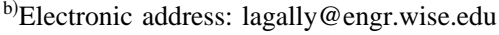

we explore an alternative approach, spontaneous selfembedding of high-Ge-concentration SiGe dome islands in a conformal shell of low-concentration SiGe alloy, which can be followed by Si deposition to recover the planar surface. The experiments employ a combination of gas-source molecular beam epitaxy (MBE), low-energy electron microscopy (LEEM), and cross-sectional transmission electron microscopy (XTEM). They exploit the Stranski-Krastanov nature of the growth of SiGe on $\mathrm{Si}(100)$. The growth starts with the formation of a 2D SiGe wetting layer, whose thickness depends on the Ge concentration, followed by a first phase of 3D islands-small pyramidal huts, ${ }^{11}$ which, upon reaching a well-defined critical volume, undergo a shape transition into steeper-faceted domes. ${ }^{12,13}$ The concept of self-embedding is based on the idea that once domes are formed it becomes energetically more favorable for SiGe alloy material to be incorporated in these islands than to remain in a $2 \mathrm{D}$ wetting layer. By growing a low-concentration alloy wetting layer prior to the formation of islands with higher Ge concentration, we can create a large reservoir of SiGe alloy with well-defined composition. The transition to dome shape then initiates the spontaneous coating of the island surface with material from this reservoir. As a result of the abrupt nature of this spontaneous process, intermixing and shape changes are kinetically suppressed and the highconcentration $\mathrm{SiGe}$ dome islands retain their shape.

The samples were grown by gas source $\mathrm{MBE}$ at $750{ }^{\circ} \mathrm{C}$ on $\mathrm{Si}(100)$ substrates using $\mathrm{Si}_{2} \mathrm{H}_{6}$ and $\mathrm{Ge}_{2} \mathrm{H}_{6}$. Following substrate cleaning, a low-concentration $\mathrm{Si}_{0.85} \mathrm{Ge}_{0.15}$ alloy wetting layer with a thickness ( $\sim 95$ monolayers, ML) close to the onset of 3D-island formation was deposited. The Ge concentration was then increased to about $40 \%$ to drive the rapid nucleation of a dense array of 3D hut islands (after the formation of a second, high-Ge-concentration 2D wetting layer much thinner than the low-Ge-concentration wetting layer). In one set of samples the growth was interrupted after this array of huts was formed ( $\sim 9.5 \mathrm{ML})$ nominal high-Geconcentration alloy layer thickness). In another, the growth was continued until the shape transition from hut to dome occurred (about 20 ML nominal high-Ge-concentration alloy layer thickness). The growth was monitored in real time by LEEM. ${ }^{9,10}$ The structure of the islands was then investigated ex situ by XTEM using a Philips CM200 microscope at 200 $\mathrm{kV}$. The TEM samples were thinned to an average thickness 


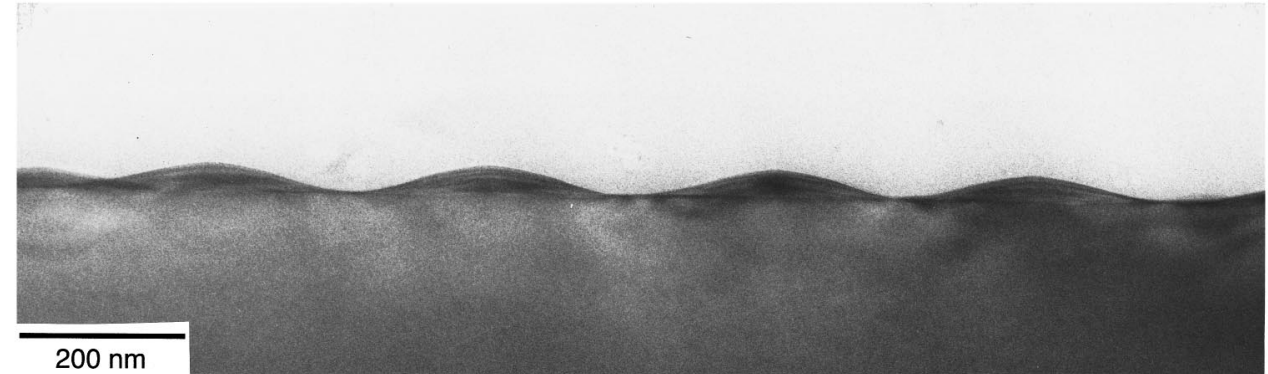

FIG. 1. Bright-field [110]-crosssection TEM micrograph of a film in which pyramidal SiGe huts have undergone the shape transition and been embedded by low-concentration SiGe alloy layer that was originally in a 2D wetting layer. of the order of $20 \mathrm{~nm}$ by tripod polishing followed by brief ion milling.

Figure 1 is a [110]-cross-section bright-field TEM micrograph of a film grown in the above manner past the point at which the transition of the islands from huts to domes has occurred. It shows a closely packed string of 3D dome islands that are identical in size and shape. Careful analysis indicates that they are bounded by straight segments corresponding to shallow $11^{\circ}\{105\}$ facets near the apex and by steeper segments corresponding to $24^{\circ}$ facets near the base. ${ }^{14}$ Despite the prior growth of a thick wetting layer with low $\mathrm{Ge}$ concentration, the dome islands appear to rest directly on the Si substrate, an observation most easily made between the islands. In addition, the contrast within the islands is not uniform: there are regions with bright and dark contrast, with well-defined boundaries.

Figure 2 gives a detailed comparison of islands in the two types of films we have grown. Figure 2(a) shows the film morphology resulting if growth is interrupted shortly before the onset of the transition from hut to dome, preserving pyramid-shaped hut islands (well-defined $11^{\circ}$ facets). Contrast is homogeneous throughout these pyramidal islands. The low-Ge-concentration wetting layer, with a thickness of $13 \mathrm{~nm}$, as well as the much thinner high-Geconcentration layer on top of it, are clearly visible. For the film shown in Fig. 2(b) the growth was stopped immediately after the transition of the island shape from pyramidal to
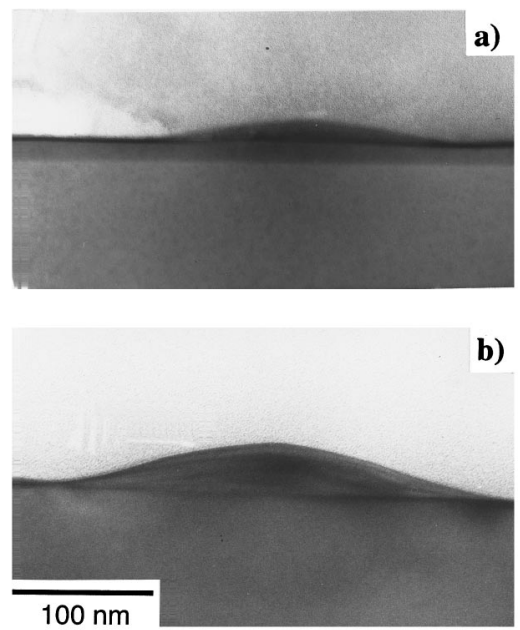

FIG. 2. Bright-field [110] cross-section micrographs (with equal scaling) showing 3D SiGe islands for films in which the growth was interrupted (a) shortly before the transition from pyramidal to dome-shaped islands and (b) immediately thereafter. In both films first $\sim 95 \mathrm{ML}$ of low-concentration $\mathrm{Si}_{0.85} \mathrm{Ge}_{0.15}$ alloy were deposited, followed by the growth of high-Geconcentration $\mathrm{Si}_{0.60} \mathrm{Ge}_{0.40}$ alloy with nominal thickness of $\sim 9.5 \mathrm{ML}$ for the

film shown in (a) and $\sim 20 \mathrm{ML}$ for the film in (b). band-edge luminescence in the Si substrate. The dominant
Downloaded $22 \mathrm{Feb} 2007$ to 128.104.198.190. Redistribution subject to AlP license or copyright, see http://apl.aip.org/apl/copyright.jsp dome-like took place, i.e., after additional deposition of high-Ge-concentration alloy material with nominal thickness of $\sim 10 \mathrm{ML}$. After the transformation into domes the thick low-Ge-concentration alloy layer is no longer observed in the regions between islands (see Fig. 1). The contrast within the domes is no longer uniform. A dark core is embedded in a shell of light contrast. ${ }^{15}$ In other words, the pyramids [Fig. 2(a) ] consist of a homogeneous SiGe alloy, while the domes [Fig. 2(b)] have a core of high Ge concentration embedded in a lower-Ge-concentration alloy matrix. The lighter regions at the base of the dome islands presumably correspond to the initial low-Ge-concentration wetting layer, while the lightercontrast coating on the dome can only be explained by assuming mass transfer from the free-standing wetting layer reservoir between the islands.

The micrographs of Fig. 2 show that in the films in which the growth was interrupted shortly before the shape transition from a $\{105\}$ faceted pyramidal hut to a multifaceted dome, the low-Ge-concentration alloy wetting layer is preserved and the pyramidal islands sit on top of it. Obviously, the energetically favorable configuration is an island plus wetting layer. The domes, which over part of their surface have steeper bounding facets, can relieve strain much more efficiently. The shape transition is therefore accompanied by an abrupt drop in the chemical potential of the island, ${ }^{12}$ and it becomes energetically favorable even for the low-Ge-concentration alloy in the thick wetting layer between the islands to be incorporated into the dome-shaped islands. As a result the low-Ge-concentration wetting layer coats the high-Ge-concentration island. We believe that the abrupt nature of the shape transition drives a rapid redistribution of material onto the island surface, mimicking growth of this cap layer at a very high deposition rate. Intermixing with the material in the core is thus kinetically limited even for the high growth temperatures used here, and the shape of the embedded (dome) island should remain unchanged. This result is in sharp contrast to embedding of hut or dome islands under ordinary growth conditions, for which a significant change in the island shape is observed..$^{6,8-10}$

We have used photoluminescence (PL) spectroscopy on embedded and uncapped islands to demonstrate that selfembedding produces efficient surface passivation. The PL measurements were performed at $T=15 \mathrm{~K}$, using an $\mathrm{Ar}^{+}$ laser at $\lambda=514.5 \mathrm{~nm}$ with a power density of $50 \mathrm{~mW} / \mathrm{mm}^{2}$. The measurements were performed in lock-in mode using a liquid-nitrogen cooled Ge detector. Figure 3 shows the spectra from the films whose morphology is shown in Figs. 2(a) and 2(b). The peak at $1.1 \mathrm{eV}$ is due to the phonon assisted band-edge luminescence in the $\mathrm{Si}$ substrate. The dominant 


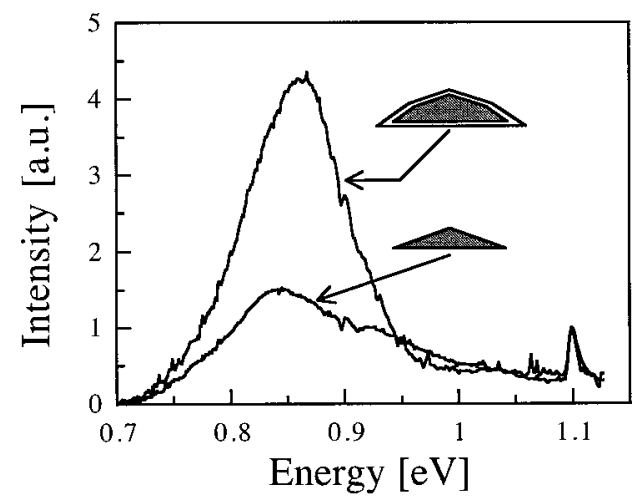

FIG. 3. Photoluminescence spectra of films with pyramidal islands [morphology in Fig. 2(a)] and with spontaneously self-embedded islands [morphology shown in Fig. 2(b)], measured at $15 \mathrm{~K}$.

feature in both spectra is the luminescence band centered around $0.85 \mathrm{eV}$, which originates from the 3D islands. ${ }^{16} \mathrm{Py}-$ ramidal huts and dome shaped islands give a very similar energy of this band, with the luminescence from pyramids slightly red shifted with respect to the one from domes. Experiments on uncapped 3D islands after exposure to air show either no island-related PL band ${ }^{17}$ or a significant reduction in intensity and a red shift of this band relative to the one in as-grown samples, which is attributed to oxidation. ${ }^{18}$ While such uncontrolled oxidation is expected to occur for the uncapped huts, a stable oxide should form on the low-Geconcentration (i.e., high $\mathrm{Si}$ concentration) shell of the selfembedded domes. One would thus expect an intense and well-defined PL band in the case of embedded dome-shaped islands, and a significantly diminished PL intensity and red shift for uncapped pyramids. Figure 3 shows exactly this qualitative behavior, and thus demonstrates the enhanced surface passivation of the self-embedded domes.

In conclusion, we have demonstrated the spontaneous self-embedding of dome-shaped $\mathrm{SiGe}$ islands in a lowconcentration SiGe alloy, which we propose as a first stage of a novel concept for epitaxial embedding of SiGe dome islands (or islands in any other system that have formed as a consequence of a rapid shape transformation) while preserv- ing to a great extent their original shape. The driving force for the self-embedding is believed to be the rapid drop in the chemical potential of the islands accompanying the shape transition from pyramid to dome. The efficiency of the surface passivation provided by the spontaneous Si-rich coating of the dome surfaces is clearly shown by PL measurements.

This work was supported by ONR and AFOSR.

${ }^{1}$ J. Tersoff, C. Teichert, and M. G. Lagally, Phys. Rev. Lett. 76, 1675 (1996); C. Teichert, M. G. Lagally, L. J. Peticolas, J. C. Bean, and J. Tersoff, Phys. Rev. B 53, 16334 (1996).

${ }^{2}$ D. Bimberg, M. Grundmann, and N. N. Ledentsov, MRS Bull. 23, 31 (1998), and references therein.

${ }^{3}$ Q. Xie, A. Madhukar, P. Chen, and N. P. Kobayashi, Phys. Rev. Lett. 75, 2542 (1995).

${ }^{4}$ B. Rahmati, W. Jaeger, H. Trinkaus, R. Loo, L. Vescan, and H. Lüth, Appl. Phys. A: Solids Surf. 62, 575 (1996).

${ }^{5}$ A. A. Darhuber, P. Schittenhelm, V. Holy, J. Stangl, G. Bauer, and G. Abstreiter, Phys. Rev. B 55, 15652 (1997); P. Schittenhelm, G. Abstreiter, A. Darhuber, G. Bauer, P. Werner, and A. Kosogov, Thin Solid Films 294, 291 (1997).

${ }^{6}$ E. Mateeva, P. Sutter, J. C. Bean, and M. G. Lagally, Appl. Phys. Lett. 71, 3233 (1997).

${ }^{7}$ A. Zunger, MRS Bull. 23, 15 (1998).

${ }^{8}$ P. Sutter, E. Mateeva, and M. G. Lagally, J. Vac. Sci. Technol. B 16, 1560 (1998).

${ }^{9}$ P. Sutter and M. G. Lagally, Phys. Rev. Lett. 81, 3471 (1998).

${ }^{10}$ P. Sutter, E. Mateeva, J. S. Sullivan, and M. G. Lagally, Thin Solid Films (to be published).

${ }^{11}$ Y.-W. Mo, D. E. Savage, B. S. Swarzentruber, and M. G. Lagally, Phys. Rev. Lett. 65, 1020 (1990).

${ }^{12}$ F. M. Ross, J. Tersoff, and R. M. Tromp, Phys. Rev. Lett. 80, 984 (1998).

${ }^{13}$ G. Medeiros-Ribeiro, A. M. Bratkovski, T. I. Kamins, D. A. A. Ohlberg, and R. S. Williams, Science 279, 353 (1998).

${ }^{14}$ Because the XTEM images are [110] cuts through the islands, the observed base length corresponds to the diagonal of their $\langle 100\rangle$ aligned bases. The corresponding facet angles are $8^{\circ}$ and $17^{\circ}$.

${ }^{15}$ Insofar as mass-thickness contrast is mainly responsible for image formation in bright-field imaging, the regions of darker contrast correspond to $\mathrm{SiGe}$ alloy with higher Ge concentration.

${ }^{16}$ H. Sunamura, N. Usami, Y. Shiraki, and S. Fukatsu, Appl. Phys. Lett. 66, 3024 (1995); P. Schittenhelm, M. Gail, J. Brunner, J. F. Nuetzel, and G. Abstreiter, ibid. 67, 1292 (1995); H. Sunamura, Y. Shiraki, and S. Fukatsu, ibid. 66, 953 (1995); R. Apetz, L. Vescan, A. Hartmann, C. Dieker, and H. Lüth, ibid. 66, 445 (1995).

${ }^{17}$ L. Vescan (private communication).

${ }^{18}$ Y. Kishimoto, Y. Shiraki, and S. Fukatsu, Appl. Phys. Lett. 70, 2837 (1997). 